\title{
James Webb Space Telescope Optical Telescope Element/Integrated Science Instrument Module (OTIS) Status
}

\author{
Lee Feinberg ${ }^{\mathrm{a}}$, Mark Voyton ${ }^{\mathrm{a}}$, Juli Lander ${ }^{\mathrm{a}}$, Ritva Keski-Kuha ${ }^{\mathrm{a}}$, Gary Matthews ${ }^{\mathrm{b}}$ \\ aNASA Goddard Space Flight Center, Greenbelt, MD; ${ }^{b}$ Harris Corporation, Rochester, NY, USA;
}

\begin{abstract}
The James Webb Space Telescope Optical Telescope Element (OTE) and Integrated Science Instrument Module (ISIM) are integrated together to form the OTIS. Once integrated, the OTIS undergoes primary mirror center of curvature optical tests, electrical and operational tests, acoustics and vibration testing at the Goddard Space Flight Center before being shipped to the Johnson Space Center for cryogenic optical testing of the OTIS. In preparation for the cryogenic optical testing, the JWST project has built a Pathfinder telescope and has completed two Optical Ground System Equipment (OGSE) cryogenic optical tests with the Pathfinder. In this paper, we will summarize optical test results to date and status the final Pathfinder test and the OTIS integration and environmental test preparations
\end{abstract}

Keywords: OTIS, OTE, ISIM space telescope

\section{INTRODUCTION}

The James Webb Space Telescope cold portion is made up of the Optical Telescope Element (OTE) and the Integrated Science Instrument (ISIM) as shown in Figure 1. Recently, both elements finished the integration and testing programs and were delivered to the next level of assembly called the OTE/ISIM (OTIS). The OTIS team is managing the integration of these two elements together and the integration has already begun at the Goddard Space Flight Center. After the ambient integration is completed, the OTIS ambient environmental tests will begin followed by the cryogenic optical testing. Prior to all of these activities the OTIS team has practiced both ambient integration and cryogenic testing using the Pathfinder telescope. Two cryogenic Pathfinder tests have already completely successfully at the Johnson Space Center (JSC) cryogenic chamber and a final third cryogenic test focused mostly on thermal testing will be performed prior to the final OTIS cryogenic test. After OTIS environmental tests are complete, the OTIS will be shipped to Northrop Grumman in Redondo Beach, California for integration with the spacecraft and sunshield and final observatory integration and testing.
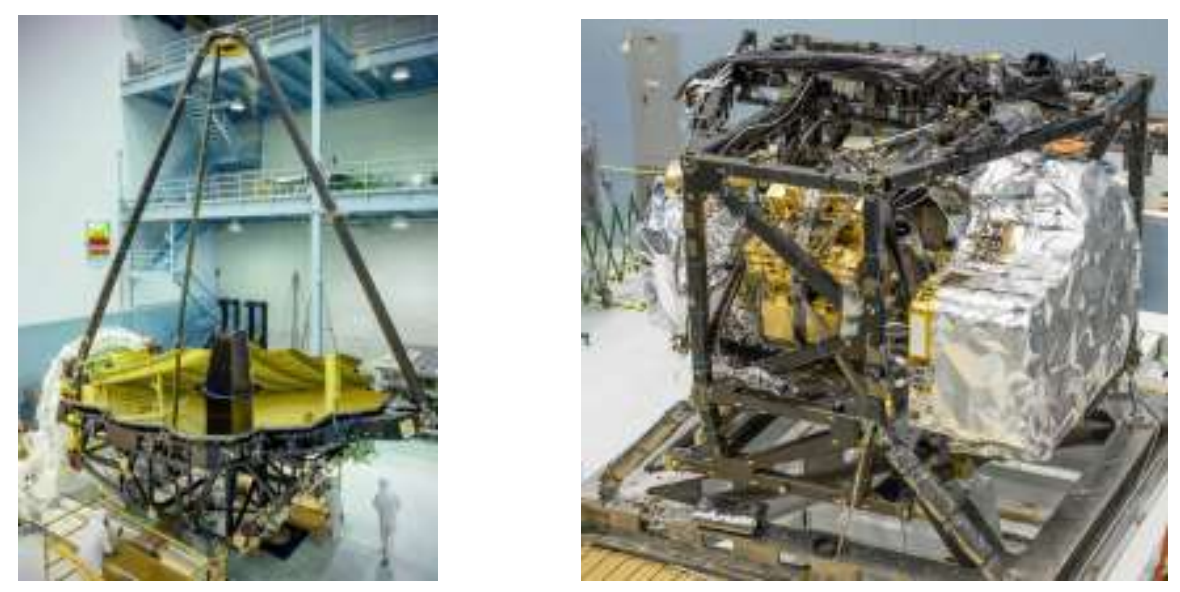

Figure 1: OTE and ISIM 
The integration and testing flow for OTIS can be seen below in Figure 2. This flow began with the development of the Ground Support Equipment (GSE) needed to perform testing which completed in the Fall of 2014. In parallel to that, the Pathfinder was built up at the Goddard Space Flight Center and shipped to the Johnson Space Center for the Optical Ground Support Equipment 1 and 2 cryogenic optical tests which occurred in 2015. The second OGSE2 test included the flight Aft Optics System (AOS). The AOS was sent back to Goddard in December 2015 for integration onto the OTE. At this point, the team is preparing for the third and final Pathfinder test called the Thermal Pathfinder test (TPF). In addition, the integration of the ISIM to OTE has begun and the ambient environmental testing preparations are well underway. At the end of the flow is the final cryogenic testing of the full flight OTIS at the JSC test facility where the Pathfinder testing occurred.

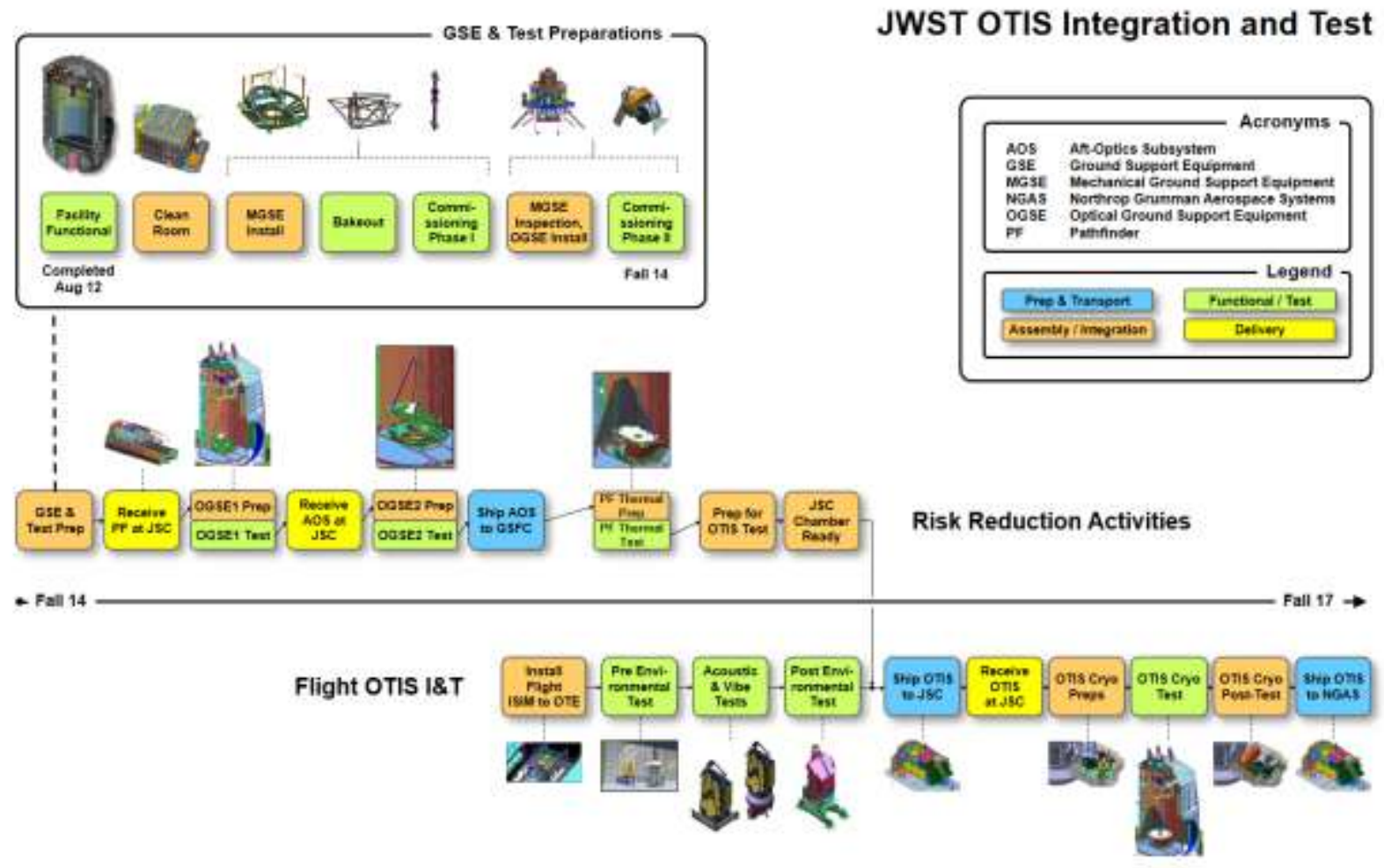

Figure 2: OTIS Integration and Testing Flow

\section{JSC TEST ARCHITECTURE}

The JSC architecture is shown below in Figure 3. The test architecture and plans for the Pathfinder testing has evolved over many years. ${ }^{\mathrm{i} i \mathrm{i}}$ At the top of the chamber is the Center of Curvature Object Assembly which holds a multiwavelength interferometer used to test the primary mirror. Four windmills on the sides of the chamber contain photogrammetry cameras used to measure alignments. In addition, Aft Optics System (AOS) Source Plate Assembly (ASPA) optical sources are installed at the Cassegrain focus of the telescope and look both downward in half pass mode and upward in pass and a half mode based on an approach that uses the science instruments as the sensors. ${ }^{\mathrm{iii}}$ These sources let us test the alignment of the AOS to the instruments as well as check the end to end optical paths using 3 autocollimating flats at the top of the chamber. The telescope sits on a Hardpoint Offloader Support Structure (HOSS) that is suspended by an isolated set of hanging rods with isolators at the top of the chamber. All of the equipment inside the 
chamber operates at helium temperatures. All of the optical Ground Support Equipment (GSE) was included in the OGSE 1 or OGSE2 tests and all have now been checked out.

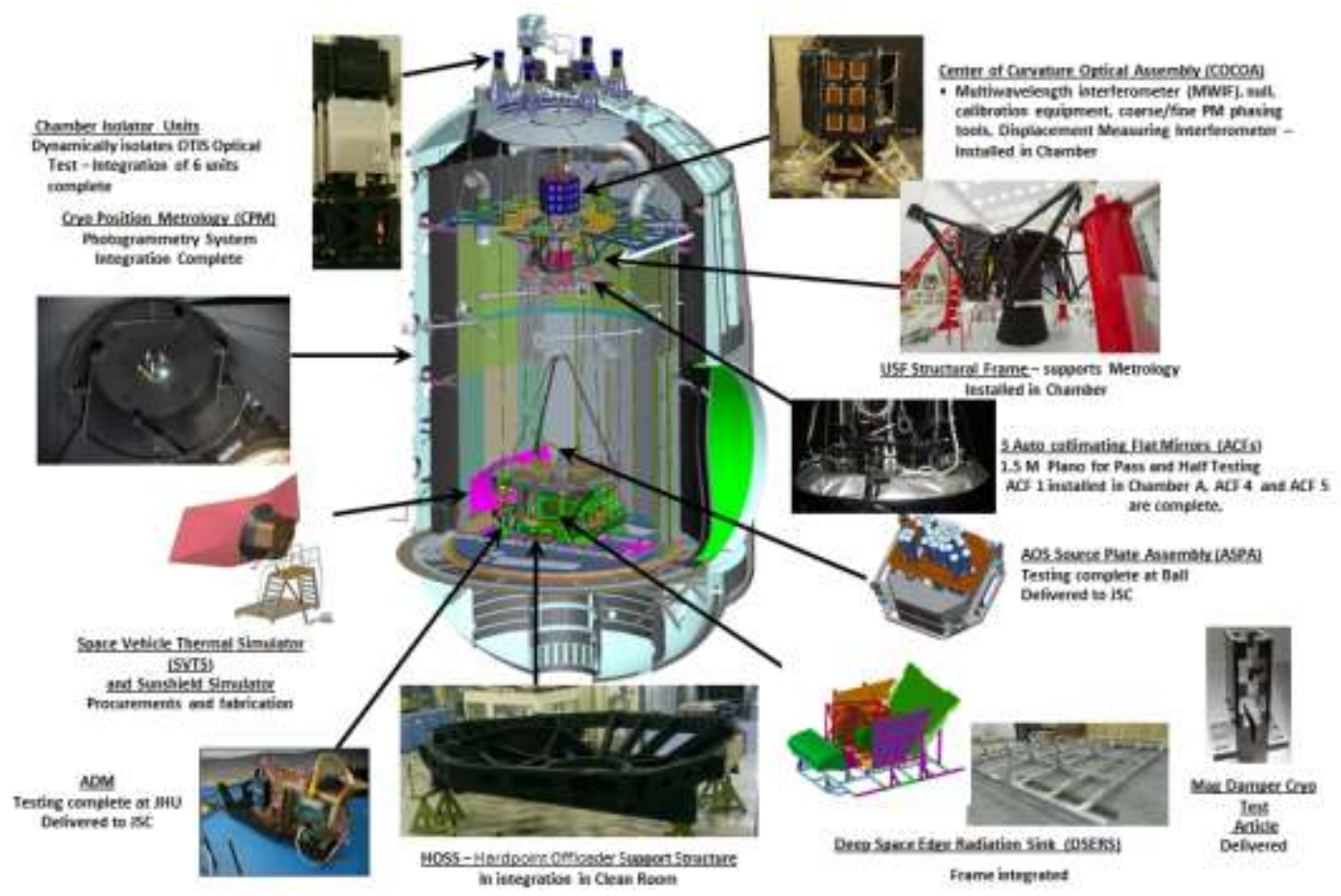

Figure 3: OTIS Test Architecture

\section{PATHFINDER TESTING AND RESULTS}

The Pathfinder telescope is used in three different configurations on three different cryogenic tests. During the OGSE1 pathfinder test, the pathfinder shown in Figure 4 includes 2 primary mirror segment spares and a secondary mirror. The inner or A segment is gold coated and completely finished while the B or outer segment is a partially polished bare beryllium mirror. This configuration allowed the test team to check the performance of all optical tests except those involving the ASPA sources. During the OGSE2 test, the AOS was installed on the pathfinder and the ASPA was placed on the AOS. A ground support Beam Image Analyzer that used an infrared detector and translation stages was used to simulate the infrared instruments so both half pass and pass and a half testing could be conducted.

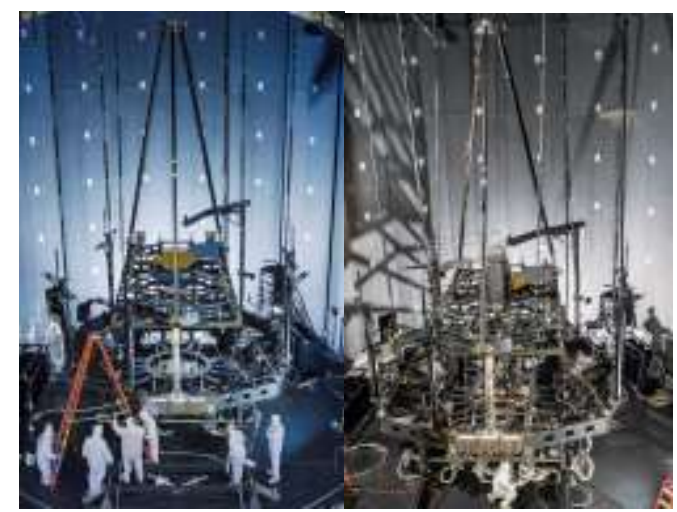

Figure 4: Pathfinder in OGSE1 (left) and OGSE2 (right) 
The optical testing during OGSE 1 and 2 was extremely valuable in checkout of Ground Support Equipment. During OGSE1, mandatory objectives were met including performing a cryogenic proof load test of the Aft Optics System (AOS) interface to assure OGSE2 can proceed safely. The team demonstrated Multi-wavelength High Speed Interferometry of the primary mirror including successfully phasing two primary mirror segments and performed detailed dynamics characterization of the isolation system. The team performed functional testing and characterized the Beam Image Analyzer and showed that it was aligned well enough for OGSE2. In addition, the photogrammetry worked extremely well including implementing lessons learned from the Cryogenic Commissioning Test. Important lessons learned including identifying a mechanical short to ground that happened during cooldown which was corrected prior to OGSE2.

OGSE2 was also an extremely valuable test. All Primary, Secondary, Tertiary Test Objectives were all met including demonstrating every optical test at some level. During this test the team was able to go through the entire primary mirror alignment sequence shown in Figure 5. During OGSE1 and then OGSE2, the team got the first vertical gravity orientation measurements of a flight mirror segment and subsequent analysis shown below in Figure 6 shows excellent correlation to the models. The team also got high quality data in the half pass and pass and a half test and examples of this are shown in Figure 7. The team did identify higher than expected vibration in pass and a half results resulting from connections to the chamber that are being mitigated. In addition, the team developed an alternative way to measure alignment in the pass and a half test using a Hartmann type approach ${ }^{\text {iv }}$. The combination of future improvements in dynamics isolation with the optical Hartmann approach will make future testing very robust.

\section{Coarse Alignment}

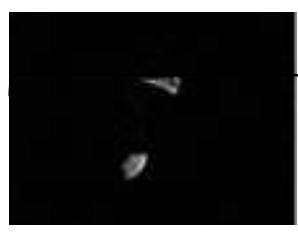

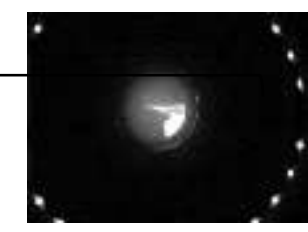

Fine Alignment

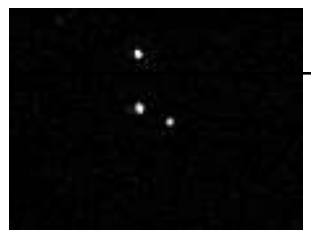

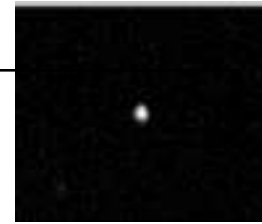

\section{Multi-Wavelength Phasing Using Synthetic Wavelengths}

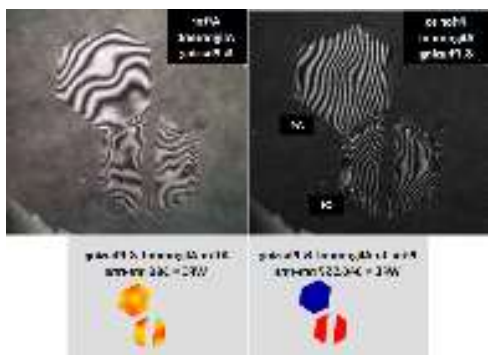

Figure 5: Primary Mirror Alignment

Measured (165 nm-rms) Model Predict (161 nm-rms) Difference (31 nm-rms)

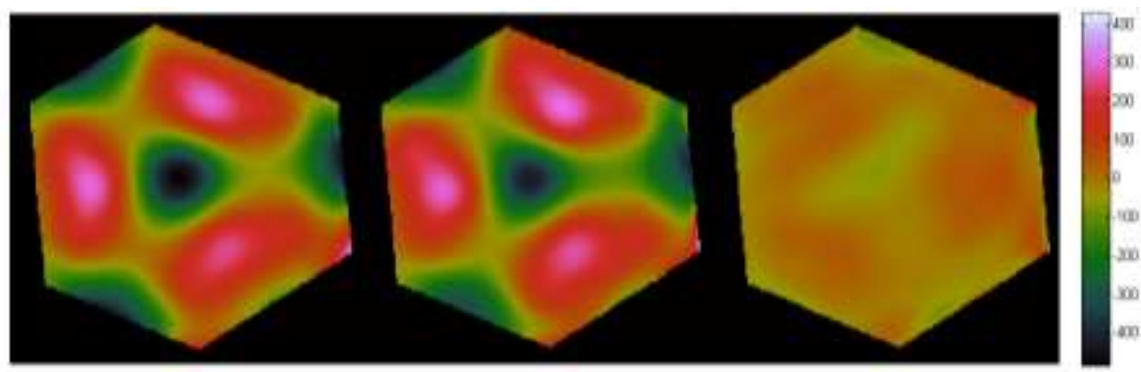

Figure 6: Vertical Gravity Error Measured vs. Prediction 

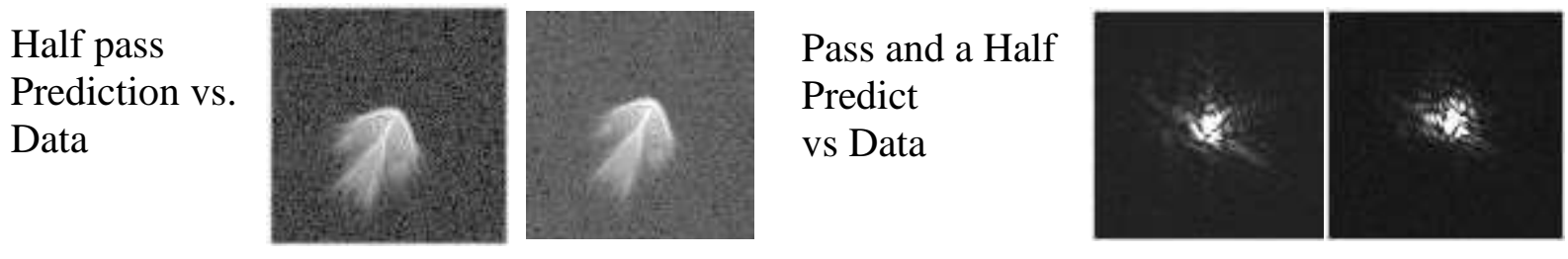

Figure 7: Half pass and Pass and a Half Test Results

In figure 8, we summarize all of the optical tests, their priorities, and when they were demonstrated. The final column shows only 3 tests were impacted by the vibration but those are being mitigated by the Hartmann test and other improvements. Every test planned for the flight OTIS test was demonstrated during OGSE1 and OGSE2.

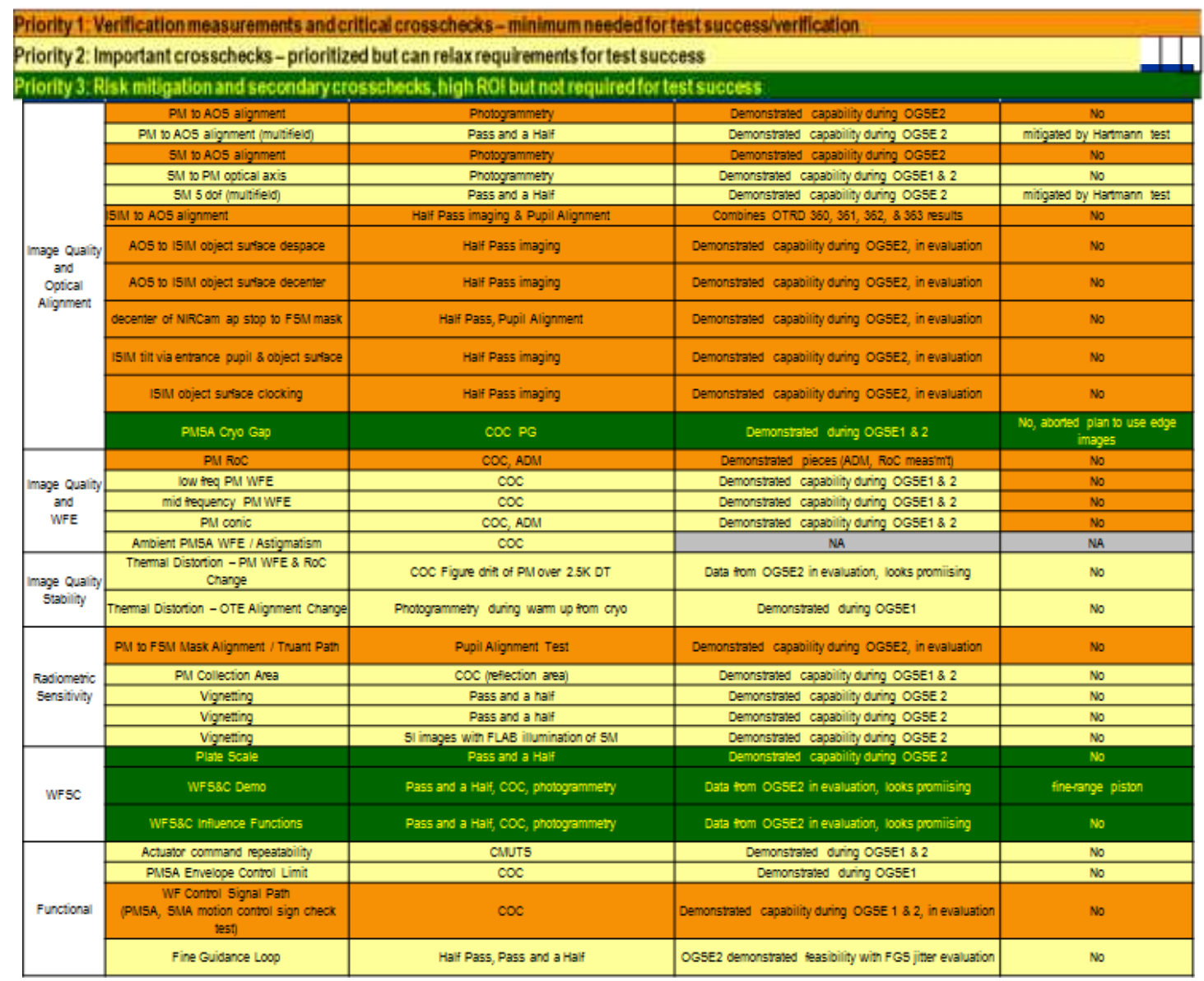

Figure 8: Optical Test matrix

The final Pathfinder test is the Thermal Pathfinder test. This test will demonstrate the thermal cooldown, warmup and thermal balance portions of the final flight OTIS test. During this test, the AOS is removed and mirror simulators are added as shown below in Figure 9. The configuration mimics the flight thermal configuration as much as possible for practice purposes. 


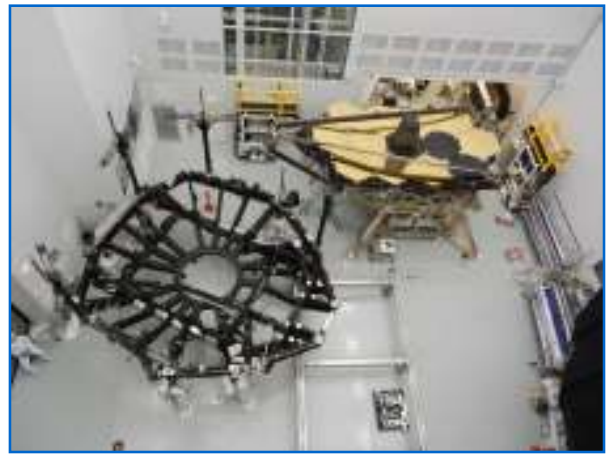

Figure 9: Thermal Pathfinder Mirror Simulators Installed

\section{AMBIENT INTEGRATION AT GSFC}

The flow of the ambient integration and testing program that occurs at Goddard is shown below in Figure 10. The first phase is to complete installation of the Fixed ISIM radiators followed by installation of the ISIM itself. Following that, the Aft Deployable ISIM Radiators (ADIR) and the ISIM Electronics Compartment (IEC) and Harness Radiator (HR) are all installed. At that point the full OTIS is assembled and ready for its environmental test program. The environmental testing includes pre and post system functional, center of curvature optical tests of primary mirror segments, alignment checks, and gap measurements. The actual environmental tests include both acoustics and vibration tests. After this all is complete, the OTIS is prepared for shipment to JSC for the final OTIS cryogenic test.

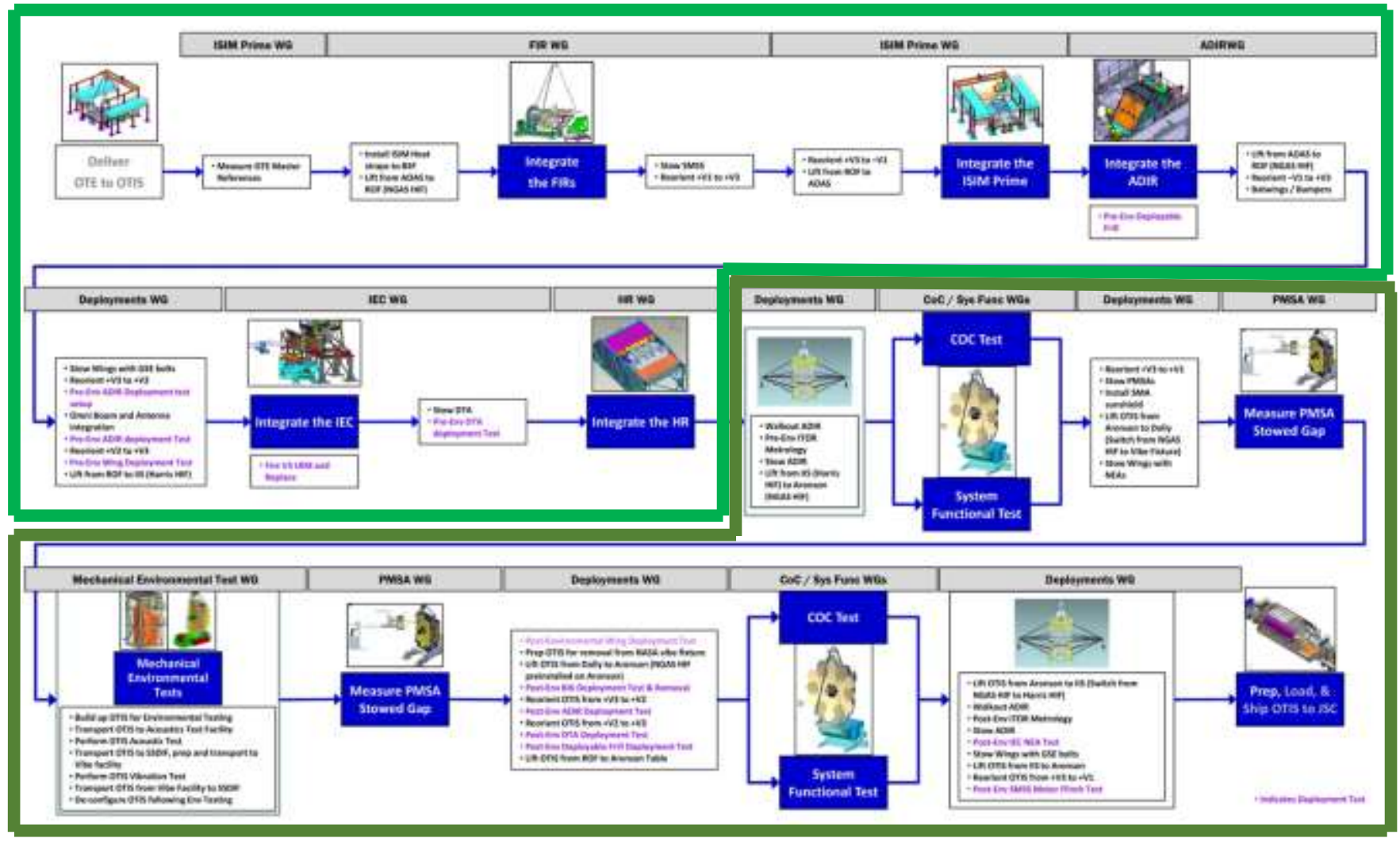

Figure 10: Ambient Integration and Test Flow 
The critical aspects of the environmental test program will be the testing of the primary mirror segment assemblies which will be seeing vibro-acoustics while attached to the wings. To assure mirrors do not change figure, static wavefront measurements will be measured of each mirror pre and post testing using the configuration shown in the left of Figure 11. In addition, the entire OTIS will undergo vibration and acoustics testing while in a large clean tent as shown on the right side of Figure 11. In addition to static wavefront measurements, a stinger will be used during optical testing attached to the backplane which will provide dynamics information on each segment using techniques developed for this test that provide both diagnostic information and model crosscheck information. ${ }^{\mathrm{v}}$

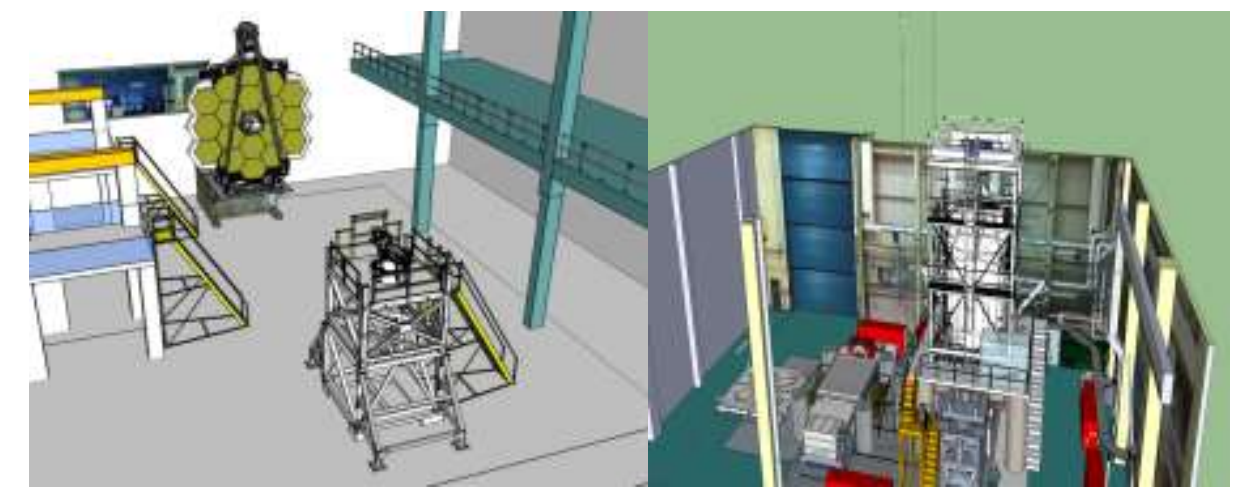

Figure 11: Center of Curvature Test Configuration (left) and Acoustics/Vibe Table with Tent (Right)

\section{CONCLUSION}

At this point, the OTIS efforts are already well underway. The Pathfinder ambient and cryogenic optical testing has been incredibly valuable and thanks to the Pathfinder testing all optical tests have been checked out. The improvements to reduce the JSC test vibration levels will be implemented in time for the Thermal pathfinder test so that they can be checked out. The new Hartmann test approach is being practiced on the Ball testbed telescope. The ambient integration is well underway and progress is being made on test facilities and test hardware.

\section{REFERENCES}

\footnotetext{
${ }^{i}$ New Approach to Cryogenic Optical Testing the James Webb Space Telescope, L. Feinberg, J. Hagopian, C. Diaz, Proceedings of SPIE, 2006

${ }^{i i}$ Use of a Pathfinder Optical Telescope Element for James Webb Space Telescope Risk Mitigation, L. Feinberg, R. Keski-Kuha, S. Texter, C. Atkinson

iii James Webb Space Telescope Cryogenic Optical Test Plans, L. Feinberg, A. Barto, M. Waldman, T. Whitman, Proceeding of SPIE,

${ }^{\text {iv }}$ Hartmann Test for the James Webb Space Telescope, S. Knight, L. Feinberg, J. Howard, S. Acton, T. Whitman, K. Smith, Proceeding of SPIE, 2016

${ }^{v}$ Nanometer Level Characterization of the JWST Optomechanical Systems Using High Speed Interferometry, B. Saif, D. Chaney, et al, Applied Optics, Vol 54, No 13, May 12015
} 